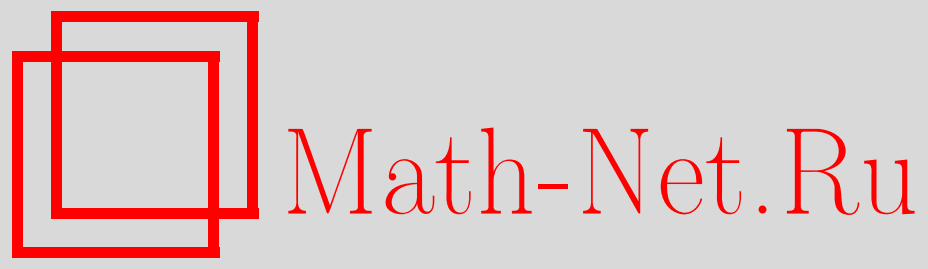

Б. Л. Фейгин, Коммутативные вертексные алгебры и их вырождения, Функи. анализ и его прил., 2014, том 48, выпуск 3, 24-33

DOI: https://doi.org/10.4213/faa3156

Использование Общероссийского математического портала MathNet.Ru подразумевает, что вы прочитали и согласны с пользовательским соглашением

http://www . mathnet.ru/rus/agreement

Параметры загрузки:

IP : 35.174 .16 .151

26 апреля 2023 г., 13:46:49

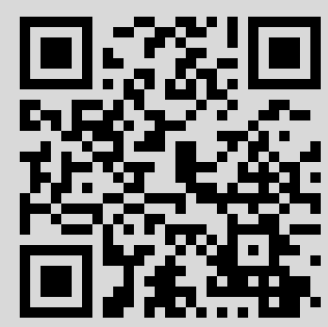




\title{
Коммутативные вертексные алгебры и их вырождения*
}

\author{
(c) 2014. Б. Л. ФЕйгин
}

Посвящается памяти И. М. Гельбанда

\begin{abstract}
Мы изучаем коммутативные вертексные алгебры, которые возникают как подалгебры в вертексных алгебрах, отвечающих алгебрам Каца-Муди. Мы описываем системы определяющих соотношений, а также вырождения в алгебры с квадратичными соотношениями. Полученные результаты могут быть использованы для получения фермионных формул для характеров.
\end{abstract}

\section{Введение}

Пусть $A$ - коммутативная алгебра. С ней можно связать коммутативную вертексную алгебру $\mathscr{O}_{A}$, ее образующие - токи $c(z), c \in A$, а операторное произведение имеет вид $c_{1}(z) c_{2}(w)=c_{1} c_{2}(z)+\cdots$. Например, если $A-$ одномерная алгебра с тривиальным умножением, то $\mathscr{O}_{A}-$ алгебра с одним генератором $c(z)$ и определяющим соотношением $c(z)^{2}=0$. Алгебры $\mathscr{O}_{A}$ естественно возникают при изучении интегрируемых представлений аффинных алгебр. Скажем, если взять в алгебре Ли $\mathfrak{s l}_{2}$ нильпотентный элемент $е$ и образовать ток $e(z)$ в $\widehat{\mathfrak{s l}}_{2}$, то в интегрируемом представлении уровня $k$ ток $e(z)^{k+1}$ действует нулем. Более точно: в вертексной алгебре, отвечающей аффинной алгебре $\widehat{\mathfrak{s l}}_{2}$ уровня $k$, содержится абелева подалгебра $\mathscr{O}_{A}$, где $A=t \mathbb{C}[t] /\left(t^{k+1}\right)$. С алгебрами Ли $\widehat{\mathfrak{s l}}_{n}$ связано много примеров. Зафиксируем в $\mathfrak{s l}_{n}$ абелеву подалгебру $\left\{E_{1, s}\right\}_{2 \leqslant s \leqslant n}$, где $E_{1, s}-$ стандартные матричные единицы в $\mathfrak{s l}_{n}$. Пусть $a_{i}(z)-$ ток, соответствующий элементу $E_{1, i+1}$. В интегрируемых $\widehat{\mathfrak{s l}}_{n}$-модулях уровня $k$ токи $a_{1}(z), \ldots, a_{n-1}(z)$ удовлетворяют соотношениям $a_{1}(z)^{\alpha_{1}} \cdots a_{n-1}(z)^{\alpha_{n-1}}=0$ для всех наборов $\alpha_{1}, \ldots, \alpha_{n-1}$, таких, что $\sum \alpha_{i}=k+1$. Можно показать, что вертексная алгебра $\widehat{\mathfrak{s l}}_{n}$ уровня $k$ содержит подалгебру $\mathscr{O}_{A}, A=\mathbb{C}\left[t_{1}, \ldots, t_{n-1}\right]_{+} / J_{k+1}$, где $\mathbb{C}\left[t_{1}, \ldots, t_{n-1}\right]_{+}$- алгебра полиномов без свободного члена, а $J_{k+1}$ - идеал, порожденный одночленами $t_{1}^{\alpha_{1}} \cdots t_{n-1}^{\alpha_{n-1}}, \sum \alpha_{i}=k+1$.

Другой источник абелевых вертексных алгебр - процедура абелинизации, или вырождения, которую можно применить к неабелевым вертексным алгебрам. Наиболее известный пример (см. [4]): возьмем вертексную алгебру $\mathscr{O}$, отвечающую минимальной модели алгебры Вирасоро типа $(2,2 p+1)$. На $\mathscr{O}$ введем фильтрацию Пуанкаре-Биркгофа-Витта, положив, что генератор $T(z)$, отвечающий алгебре Вирасоро, имеет фильтрацию 1. Тогда присоединенная градуированная вертексная алгебра абелева и совпадает с $\mathscr{O}_{A}$, где $A=t \mathbb{C}[t] /\left(t^{p+1}\right)$. Несколько менее известный пример: возьмем минимальную модель алгебры Ви-

*Работа была частична поддержана грантами РФФИ 13-01-12401-офи_м, 12-01-00836-а. Исследование осуществлено в рамках Программы «Научный фонд НИУ ВШ̈Э» в 2013-2014 гг., проект №12-01-0016. 
расоро типа $(3,10)$. Тогда соответствующую вертексную алгебру можно расширить примарным полем $\varphi_{1,9}$ (это последнее поле в таблице Каца). На полученной алгебре можно ввести фильтрацию, так что присоединенная градуированная алгебра есть $\mathscr{O}_{A}$, где $A=\mathbb{C}\left[t_{1}, t_{2}\right]_{+} /\left(t_{1}^{2}, t_{1} t_{2}, t_{2}^{3}\right)$. Аналогичное утверждение справедливо для минимальных моделей типа $(3, p)$. Если взять вертексную алгебру $\widehat{\mathfrak{s l}}_{2}$ уровня 1 и построить по ней присоединенную градуированную по фильтрации Пуанкаре-Биркгофа-Витта, то получится $\mathscr{O}_{A}$, где $A=\mathbb{C}\left[t_{1}, t_{2}, t_{3}\right]_{+} / J$, а $J$ порожден пятью квадратичными элементами. (Отождествим $\mathbb{C}\left[t_{1}, t_{2}, t_{3}\right]$ с симметрической алгеброй от $\mathfrak{s l}_{2}, S^{2}\left(\mathfrak{s l}_{2}\right)$ - сумма 5-мерного и 1-мерного представлений алгебры $\mathfrak{s l}_{2}$ и идеал $J$ порожден пятимерной компонентой.)

K сожалению, не существует регулярных способов изучать абелинизации, каждый случай приходится рассматривать отдельно. Примеры показывают, что в ряде случаев получается алгебра вида $\mathscr{O}_{A}$, а, стало быть, характеры некоторых представлений таких алгебр обладают хорошими модулярными свойствами. В общем случае не видно никаких причин, почему характеры абелевых вертексных алгебр должны быть модулярными функциями или формами.

Характеры представлений алгебр $\mathscr{O}_{A}$ вычислять трудно, пожалуй, единственный известный способ - выродить алгебру $\mathscr{O}_{A}$ в абелеву вертексную алгебру с квадратичными соотношениями, а для характеров представлений алгебр с квадратичными соотношениями имеется явная (правда, довольно сложная) формула, которая называется формулой Гордона (см. [2], [3]).

В этой работе обобщается феномен, найденный в [7], где изучалось действие абелевой подалгебры $\left\{E_{1,2}(z), E_{2,3}(z)\right\}$ алгебры $\widehat{\mathfrak{s l}}_{3}$ в интегрируемых представлениях этой алгебры $\widehat{\mathfrak{s l}}_{3}$. Наряду с прочими были получены следующие результаты. Пусть $A_{k}=\mathbb{C}\left[t_{1}, t_{2}\right]_{+} /\left(t_{1}^{\alpha} t_{2}^{\beta}, \alpha+\beta=k+1\right)$. Алгебра, порожденная подалгеброй $\left\{E_{1,2}(z), E_{2,3}(z)\right\}$ в $\mathrm{U}\left(\widehat{\mathfrak{s l}}_{3}\right)$ уровня $k$, изоморфна $\mathscr{O}_{A_{k}}$. Существует плоское семейство абелевых вертексных алгебр $\mathscr{O}_{A_{k}}[\hbar]$, которые при $\hbar \neq 0$ все изоморфны $\mathscr{O}_{A_{k}}$, а при $\hbar=0$ получается коммутативная алгебра с генераторами $b_{1}(z), \ldots, b_{k}(z)$ и $c_{1}(z), \ldots, c_{k}(z)$ и определяющими квадратичными соотношениями

$$
\begin{gathered}
b_{\alpha}^{(i)}(z) b_{\beta}(z)=c_{\alpha}^{(i)}(z) c_{\beta}(z)=0, \quad \alpha \leqslant \beta, i<2 \alpha, \\
b_{\alpha}^{(i)}(z) c_{\beta}(z)=0, \quad i<\alpha+\beta-k
\end{gathered}
$$

(здесь верхний индекс $(i)$ обозначает $i$-ю производную по $z$ ).

В $§ 1$ мы определяем наш основной объект: коммутативную вертексную подалгебру в $\widehat{\mathfrak{s l}}_{n}$ уровня $k$. А именно, мы указываем коммутативную подалгебру $\mathfrak{a}$ в $\mathfrak{s l}_{n}$, а затем исследуем действие $\mathfrak{a} \otimes \mathbb{C}\left[t, t^{-1}\right]$ в интегрируемых представлениях уровня $k$. Аналогичная абелева подалгебра есть в любой простой алгебре Ли $\mathfrak{g}$ типов $A, D, E$. Для того чтобы ее описать, раскрасим схему Дынкина в два цвета - черный и белый, так чтобы любое ребро соединяло вершины разного цвета. Подалгебра натянута на векторы, отвечающие положительным простым корням белого цвета и отрицательным простым корням черного цвета. Мы исследуем случай $\mathfrak{s l}_{n}$, но в конце второго параграфа мы покажем, что наши методы работают и в более общем случае. В 11 мы описываем абелеву вертексную подалгебру, порожденную алгеброй $\mathfrak{a} \otimes \mathbb{C}\left[t, t^{-1}\right]$ в вертексной алгебре $\widehat{\mathfrak{s l}}_{n}$ уровня $k$, т. е. явно указываем определяющие соотношения (теорема 1.1). В теореме 1.3 
мы приводим абелеву вертексную алгебру с квадратичными соотношениями, в которую нашу алгебру можно выродить. В конце §1 мы указываем некоторую абелеву подалгебру в вертексной алгебре, отвечающей тороидальной алгебре $\mathfrak{s l}_{n}$, и формулируем аналоги теорем 1.1 и 1.3 для нее. В $\S 2$ содержится схема доказательства теорем 1.1 и 1.3, а также приводятся примеры алгебр, для которых аналоги теорем 1.1 и 1.3 верны. В 33 мы определяем аналог модуля Демазюра для абелевой вертексной алгебры и вычисляем его характер. Кроме того, в этом параграфе обсуждаются интересные примеры моделей демазюровского типа.

Два слова о терминологии. Вертексными алгебрами или вертекс-операторными алгебрами мы называем алгебры с ассоциативным операторным произведением, т. е. мы не требуем, чтобы в наших алгебрах содержалась алгебра Вирасоро. Токи во всех вертексных алгебрах, которые мы рассматриваем, зависят от переменной $z$ (или от $z, w$, если речь идет об операторном произведении).

\section{§1. Формулировка основных теорем}

Пусть $\mathfrak{g}$ - полупростая алгебра Ли и $\widehat{\mathfrak{g}}$ - соответствующая аффинная алгебра Каца-Муди, т. е. центральное расширение алгебры Ли $\mathfrak{g} \otimes \mathbb{C}\left[t, t^{-1}\right]$. Пусть $\mathfrak{a}$ - подалгебра в $\mathfrak{g}$. Тогда в $\widehat{\mathfrak{g}}$ имеется подалгебра $\widehat{\mathfrak{a}}$ - центральное расширение алгебры Ли $\mathfrak{a} \otimes \mathbb{C}\left[t, t^{-1}\right]$. Мы будем изучать следующий пример: $\mathfrak{g}=\mathfrak{s l}, \mathfrak{a}-$ подалгебра, порожденная матричными единицами $E_{n, n-1}, E_{n-2, n-1}, E_{n-2, n-3}, \ldots$ (т. е. отрицательный простой корень, потом положительный простой корень, потом снова отрицательный и т. д). Алгебра a состоит из нильпотентных элементов, а потому 2-коцикл алгебры $\mathfrak{s l}_{n} \otimes \mathbb{C}\left[t, t^{-1}\right]$, ограниченный на $\mathfrak{a} \otimes \mathbb{C}\left[t, t^{-1}\right]$, равен нулю. Иными словами, имеется вложение алгебры $\mathfrak{a} \otimes \mathbb{C}\left[t, t^{-1}\right]$ в $\widehat{\mathfrak{s l}}_{n}$. $\mathrm{B} \mathfrak{a} \otimes \mathbb{C}\left[t, t^{-1}\right]$ выберем стандартный базис $E_{n, n-1}[s], E_{n-2, n-1}[s], E_{n-2, n-3}[s], \ldots$, состоящий из Фурье-компонент. Для удобства изменим обозначения: положим $a_{1}[s]=E_{n, n-1}[s], a_{2}[s]=E_{n-2, n-1}[s], a_{3}[s]=E_{n-2, n-3}[s], \ldots$ Соответствующие образующим $a_{i}[s]$ токи $a_{i}(z)=\sum_{i \in \mathbb{Z}} a_{i}[s] z^{-s}$ порождают абелеву вертексную алгебру $\mathfrak{A}$. Напомним, что аффинная алгебра $\widehat{\mathfrak{s l}}_{n}$ определяет семейство вертексных алгебр $B_{k}, k \in \mathbb{C}$, где центральный элемент действует умножением на $k$. При рациональных $k>-n$ в $B_{k}$ имеется максимальный двусторонний идеал $J_{k}$. Обозначим через $B_{k}^{\text {red }}$ факторалгебру $B_{k} / J_{K}$. Обозначим через $\mathfrak{A}_{k}^{\text {red }}$ подалгебру в $B_{k}^{\text {red }}$, порожденную генераторами $a_{i}(z)$.

Теорема 1.1. Пусть $k \in \mathbb{Z}, k \geqslant 0$. Определяющие соотношения в алгебре $\mathfrak{A}_{k}^{\mathrm{red}}$ имеют вид

$$
a_{i}(z)^{x} a_{i+1}(z)^{y}=0, \quad 1 \leqslant i \leqslant n-1, x+y=k+1 .
$$

Таким образом, алгебра $\mathfrak{A}_{k}^{\text {red }}$ изоморфна алгебре $\mathscr{O}_{A}$, где

$$
A=\mathbb{C}\left[t_{1}, \ldots, t_{n}\right]_{+} /\left(t_{i}^{x} t_{i+1}^{y}, x+y=k+1, i=1, \ldots, n\right) .
$$

Вторая наша теорема описывает вырождение алгебры $\mathfrak{A}_{k}^{\text {red }}$ до квадратичной вертексной алгебры с простыми мономиальными соотношениями.

Коммутативная вертексная алгебра с квадратичными соотношениями строится по неотрицательной целочисленной квадратной симметричной $N \times N$ матрице $M=\left(m_{i, j}\right)$ и определяется как вертексная алгебра $V(M)$ с генераторами $b_{i}(z), 1 \leqslant i \leqslant N$, такая, что определяющие соотношения квадратичны 
и имеют вид $b_{i}^{(\alpha)}(z) b_{j}(z)=0$, где $b_{i}^{(\alpha)}(z)$ - производная тока $b_{i}(z)$ по $z$ порядка $\alpha$ и $\alpha<m_{i, j}$.

Замечание 1.2. Операторное произведение в такой алгебре имеет вид

$$
b_{i}(z) b_{j}(w)=\sum_{s \geqslant 0}(z-w)^{m_{i, j}+s} b_{i, j}^{s}(z) .
$$

Это означает, что в представлении этой алгебры $V(M)$ матричный элемент $\left\langle v_{1}\left|b_{i}(z) b_{j}(w)\right| v_{2}\right\rangle$ равен $(z-w)^{m_{i, j}} P(z, w)$, где $P(z, w)$ - полином Лорана. Отметим еще, что можно модифицировать определение алгебры $V(M)$ так, чтобы оно работало для любых целочисленных матриц $M$.

Теорема 1.3. Существует плоское семейство вертекс-операторнъх алгебр $\mathscr{O}[\hbar], \hbar \in \mathbb{C}$, такое, что $\mathscr{O}[\hbar] \simeq \mathfrak{A}_{k}^{\mathrm{red}}$, если $\hbar \neq 0$, a $\mathscr{O}[0]$ изоморфно квадратичной вертекс-операторной алгебре, построенной по матрище $M$, описанной ниже.

Порядок матрицы $M$ равен $(n-1) k$. Занумеруем ее строки и столбцы парами $(a, b), 1 \leqslant a \leqslant n-1,1 \leqslant b \leqslant k$. Тогда

$$
m_{(a, b),\left(a^{\prime}, b^{\prime}\right)}= \begin{cases}2 \min \left(b, b^{\prime}\right), & \text { если } a=a^{\prime}, \\ 0, & \text { если }\left|a-a^{\prime}\right|=1, b^{\prime}+b \leqslant k, \\ b^{\prime}+b-k, & \text { если }\left|a-a^{\prime}\right|=1, b^{\prime}+b \geqslant k, \\ 0, & \text { если }\left|a-a^{\prime}\right|>1,\end{cases}
$$

где $m_{(a, b),\left(a^{\prime}, b^{\prime}\right)}$ - матричный элемент в строке $(a, b)$ и столбце $\left(a^{\prime}, b^{\prime}\right)$.

Мы приведем сейчас пример абелевой вертексной алгебры, которая возникает при изучении представлений алгебры двойных токов. Рассмотрим алгебру токов на $\mathbb{C}^{*} \times \mathbb{C}$ со значениями в $\mathfrak{s l}_{n}$, т. е. $\mathfrak{s l}_{n} \otimes \mathbb{C}\left[t, t^{-1}, x\right]$. Алгебру Ли $\mathfrak{s l}_{n} \otimes \mathbb{C}\left[t, t^{-1}, x\right]$ можно центрально расширить посредством пространства $\Omega^{1}\left(\mathbb{C}^{*} \times \mathbb{C}\right) / d \Omega^{0}\left(\mathbb{C}^{*} \times \mathbb{C}\right) ; 2$-коцикл имеет вид $\omega(f, g)=\mu\langle d f, g\rangle, f, g \in \mathfrak{s l}_{n} \otimes$ $\mathbb{C}\left[t, t^{-1}, x\right]$. Здесь $\langle\cdot, \cdot\rangle$ - форма Киллинга на $\mathfrak{s l}_{n},\langle d f, g\rangle$ есть 1-форма на $\mathbb{C}^{*} \times \mathbb{C}$, а $\mu$ - проекция $\Omega^{1} \rightarrow \Omega^{1} / d \Omega^{0}$. Соответствующее коциклу $\omega$ центральное расширение называется двойной аффинной алгеброй и обозначается через $\widehat{\mathfrak{s l}_{n}}$. Нам будет нужна несколько меньшая алгебра $P \subset \widehat{\mathfrak{s l}}_{n}$, она порождена подалгебрами $\mathfrak{s l}_{n} \otimes x \mathbb{C}\left[t, t^{-1}\right]$ и $\mathfrak{s l}_{n} \otimes \mathbb{C}\left[t, t^{-1}\right]$.

Пусть $C$ - абелева алгебра, порожденная генератором $c(z)$. Существует гомоморфизм $\theta(u)$, отображающий алгебру $P$ в $\mathrm{U}\left(\mathfrak{s l}_{n}\right) \otimes C ; \theta(u)$ отображает $\mathfrak{s l}_{n} \otimes \mathbb{C}\left[t, t^{-1}\right]$ на $\widehat{\mathfrak{s l}}_{n} \otimes 1$ и ток $l(z) x \in \mathfrak{s l}_{n} \otimes x \mathbb{C}\left[t, t^{-1}\right], l \in \mathfrak{s l}_{n}$, отображается в $u l(z) \otimes c(z)$. Используя отображение $\theta(u)$, можно построить гомоморфизм $P \rightarrow B_{k}^{\mathrm{red}} \otimes C, k>0$.

Пусть $n=2 l$. Рассмотрим в $P$ абелеву подалгебру $\widehat{\widehat{a}}$. Базисом в ней служат токи $E_{1,2}(z), E_{2,3}(z), E_{3,4}(z), \ldots, E_{2 l-1,2 l}$, а также $x E_{1,2 l}(z) \in \mathfrak{s l}_{n} \otimes x \mathbb{C}\left[t, t^{-1}\right]$. Эти токи мы обозначим через $a_{1}(z), \ldots, a_{2 l}(z)$. Индексы $1, \ldots, 2 l$ отождествим с элементами группы $\mathbb{Z} / 2 l \mathbb{Z}$. Пусть $\mathfrak{P}_{k}$ - подалгебра в $B_{k}^{\text {red }} \otimes C$, порожденная образами токов $a_{i}(z)$ при гомоморфизме $\theta(u), u \neq 0$.

Теорема 1.4. (а) Алгебра $\mathfrak{P}_{k}$ коммутативна и порождена токами $a_{i}(z)$, $i \in \mathbb{Z} / 2 l \mathbb{Z}$, а определяюшие соотношения имеют вид $a_{i}(z)^{x} a_{i+1}(z)^{y}=0$ при $x+y=k+1$. 
(b) Существует плоское семейство коммутативных вертексных алгебр $\mathscr{O}[\hbar], \hbar \in \mathbb{C}$, такое, что $\mathscr{O}[\hbar] \simeq \mathfrak{P}_{k}$ при $\hbar \neq 0$, а алгебра $\mathscr{O}[0]$ изоморфна квадратичной алгебре, построенной по следующей матрице М: ее строки и столбиь занумерованы парами $(a, b), a \in \mathbb{Z} / 2 l \mathbb{Z}, 1 \leqslant b \leqslant k, u$

$$
m_{(a, b),\left(a^{\prime}, b^{\prime}\right)}= \begin{cases}2 \min \left(b, b^{\prime}\right), & \text { если } a=a^{\prime}, \\ 0, & \text { если } a-a^{\prime}= \pm 1, b^{\prime}+b \leqslant k, \\ b^{\prime}+b-k, & \text { если } a-a^{\prime}= \pm 1, b^{\prime}+b \geqslant k, \\ 0 & \text { в противном случае. }\end{cases}
$$

\section{§2. Построение вырождения в квадратичную вертексную алгебру}

Квадратичную вертексную алгебру можно реализовать как подалгебру в решетчатой алгебре. Для того чтобы это объяснить, напомним некоторые основные свойства решетчатых алгебр (см. [8]). Пусть $\mathfrak{h}$ - векторное пространство с невырожденной квадратичной формой $\langle\cdot, \cdot\rangle$. С парой $\mathfrak{h},\langle\cdot, \cdot\rangle$ свяжем вертексную алгебру (алгебру Гейзенберга) - она порождена операторами $h(z), h \in \mathfrak{h}$, а операторное произведение имеет вид $h_{1}(z) h_{2}(w)=\left\langle h_{1}, h_{2}\right\rangle(z-w)^{-2}+\cdots$. Из этого следует, что $\left[h_{1}(z), h_{2}(w)\right]=\delta^{\prime}(z / w)\left\langle h_{1}, h_{2}\right\rangle, \delta^{\prime}-$ производная дельтафункции. Алгебру Гейзенберга можно расширить, добавив вертексные операторы $p(z), p \in \mathfrak{h}$. Операторные произведения устроены так:

$h(z) p(w)=(z-w)^{-1}\langle h, p\rangle p(z)+\cdots, \quad p_{1}(z) p_{2}(w)=(z-w)^{\left\langle p_{1}, p_{2}\right\rangle}\left(p_{1}+p_{2}\right)(z)+\cdots$.

Явная формула для $p(z)$ - экспонента, ее можно найти в [8].

Пусть задана матрица $M=\left(m_{i, j}\right)$ порядка $N$. Возьмем пространство $\mathfrak{h}$ и систему $N$ линейно независимых векторов $p_{1}, \ldots, p_{N}$ в нем, таких, что $\left\langle p_{i}, p_{j}\right\rangle=m_{i, j}$.

Предложение 2.1. Вертексная алгебра, порожденная операторами $p_{i}(z)$, изоморфна квадратичной алгебре $V(M)$.

Фундаментальные представления алгебры $\widehat{\mathfrak{s l}}_{n}$ могут быть построены посредством вложения $B_{1}^{\text {red }}$ в решетчатую алгебру. Эта конструкция принадлежит Кацу и Френкелю [6] и соотносит токам из $\widehat{\mathfrak{s l}}_{n}$ вертексные операторы. При ограничении на алгебру $\widehat{\mathfrak{a}}$ мы получаем гомоморфизм $a_{i}(z) \rightarrow p_{i}(z)$, где $\left\langle p_{i}, p_{i}\right\rangle=2$, $\left\langle p_{i}, p_{i+1}\right\rangle=1$ и $\left\langle p_{i}, p_{j}\right\rangle=0$ при $|i-j|>1$. Отметим, что в конструкции КацаФренкеля имеются подкручивающие коциклы. При ограничении на $\widehat{\mathfrak{a}}$ они тривиализуются. Мы получаем доказательство теорем 1.1 и 1.3 при $k=1$. Случай большего $k$ получается при помощи тензорного произведения $k$ копий представлений уровня один.

Возьмем векторное пространство $\mathfrak{h}$ достаточно большой размерности с невырожденным скалярным произведением и систему векторов $p_{i}^{\alpha}, 1 \leqslant \alpha \leqslant k$, $1 \leqslant i \leqslant n-1$, таких, что

$$
\left\langle p_{i}^{\alpha}, p_{j}^{\beta}\right\rangle= \begin{cases}0, & \alpha \neq \beta \\ 0, & |i-j|>1 \\ 2, & \alpha=\beta, i=j \\ 1, & \alpha=\beta,|i-j|=1\end{cases}
$$

Отображение $\varphi: a_{i}(z) \rightarrow \sum_{\alpha=1}^{k} p_{i}^{\alpha}(z)$ дает вложение алгебры $\mathfrak{A}_{k}^{\text {red }}$ в решетчатую алгебру. Подкрутим теперь гомоморфизм $\varphi$, сделав его зависящим от параметра $\hbar \in \mathbb{C}:$ 


$$
\begin{aligned}
& \varphi(\hbar): a_{i}(z) \rightarrow \sum_{\alpha=1}^{k} p_{i}^{\alpha}(z) \hbar^{\alpha-1} \text { при нечетных } i, \\
& \varphi(\hbar): a_{i}(z) \rightarrow \sum_{\alpha=1}^{k} p_{i}^{\alpha}(z) \hbar^{k-\alpha} \quad \text { при четных } i .
\end{aligned}
$$

Понятно, что при $\hbar \neq 0$ отображение $\varphi(\hbar)$ является вложением алгебры $\mathfrak{A}_{k}^{\text {red }}$ в решетчатую алгебру. При $\hbar=0$ образ значительно меньше. Мы построили плоское семейство $\operatorname{Im} \varphi(\hbar)$ подалгебр в решетчатой алгебре, у этого семейства имеется предел при $\hbar=0$. Обозначим этот предел через $\mathfrak{A}_{k}^{\text {red }}(0)$. В $\mathfrak{A}_{k}^{\text {red }}(0)$, прежде всего, есть образ отображения $\varphi(0)$, т. е. $p_{1}^{1}(z), p_{2}^{k}(z), p_{3}^{1}(z), \ldots, p_{n-1}^{x}(z)$, где $x$ равно 1 или $k$ в зависимости от четности $n$. Образ оператора $a_{1}(z)^{2}$ при отображении $\varphi(0)$ равен нулю, однако

$$
\lim _{\hbar \rightarrow 0} \hbar^{-1} \varphi(\hbar)\left(a_{1}(z)^{2}\right)=p_{1}^{1}(z) p_{1}^{2}(z)=\left(p_{1}^{1}+p_{1}^{2}\right)(z) .
$$

Стало быть, оператор $\left(p_{1}^{1}+p_{1}^{2}\right)(z)$ лежит в алгебре $\mathfrak{A}_{k}^{\text {red }}(0)$. Аналогичным образом, рассматривая лидирующий член $\varphi(\hbar)\left(a_{1}(z)^{3}\right)$ при $\hbar \rightarrow 0$, находим, что оператор $\left(p_{1}^{1}+p_{1}^{2}+p_{1}^{3}\right)(z)$ лежит в алгебре $\mathfrak{A}_{k}^{\text {red }}(0)$. Проделаем тоже самое для всех токов $a_{i}(z)$. Мы установим, что в алгебре $\mathfrak{A}_{k}^{\text {red }}(0)$ имеется следующие вертексные операторы:

$$
\begin{gathered}
p_{1}^{1}(z),\left(p_{1}^{1}+p_{1}^{2}\right)(z), \ldots,\left(p_{1}^{1}+p_{1}^{2}+\cdots+p_{1}^{k}\right)(z), \\
p_{2}^{k}(z),\left(p_{2}^{k}+p_{2}^{k-1}\right)(z), \ldots,\left(p_{2}^{k}+p_{2}^{k-1}+\cdots+p_{2}^{1}\right)(z), \\
p_{3}^{1}(z),\left(p_{3}^{1}+p_{3}^{2}\right)(z), \ldots,\left(p_{3}^{1}+p_{3}^{2}+\cdots+p_{3}^{k}\right)(z),
\end{gathered}
$$

Матрица скалярных произведений этого набора векторов - в точности матрица $M$, описанная после формулировки теоремы 1.3. Поскольку алгебра, порожденная вертексными операторами, - это алгебра с квадратичными соотношениями, то для доказательства теоремы 1.3 достаточно показать, что алгебра $\mathfrak{A}_{k}^{\mathrm{red}}(0)$ порождена вертексными операторами (2.1). Покажем, как это сделать в случае $n=2$. Пусть $C$ - абелева вертекс-операторная алгебра, порожденная током $d(z)$ (без дополнительных соотношений), и пусть $D_{i}(z)=d(z)^{i}$. Токи $D_{i}(z)$ удовлетворяют очевидной системе квадратично-линейных соотношений: $D_{i}(z) D_{j}(z)=D_{i+j}(z)$. Нам понадобится более сложная система квадратичных соотношений между операторами $D_{i}(z)$, которая, разумеется, вытекает из этой более простой. Фиксируем $i, j \geqslant 1, i \geqslant j$, и положим

$$
\begin{aligned}
X_{i, j}(\varepsilon)=D_{i+j}(z+\varepsilon)-\left(\begin{array}{c}
2 j \\
1
\end{array}\right) D_{i+j-1}(z+\varepsilon) D_{1}(z) \\
\quad+\left(\begin{array}{c}
2 j \\
2
\end{array}\right) D_{i+j-2}(z+\varepsilon) D_{2}(z)-\cdots+D_{i-j}(z+\varepsilon) D_{2 j}(z) .
\end{aligned}
$$

Тогда

$$
\frac{\partial^{m} X_{i, j}}{\partial \varepsilon^{m}}=0 \text { при } m<2 j \text {. }
$$


Набор этих равенств - это соотношения между токами $D_{i}(z)$, и они являются определяющим соотношениями в алгебре $C$. При $j=1$ мы получаем два соотношения: $D_{i}(z) D_{1}(z)=D_{i+1}(z)$ и $(i+1) D_{1}^{\prime}(z) D_{i}(z)=D_{i+1}^{\prime}(z)$.

Предложение 2.2. Пусть $C_{k}$ - абелева вертекс-операторная алгебра, порожденная током $d(z)$, с определяющим соотношением $d(z)^{k+1}=0$. Тогда операторы $D_{i}(z)=d(z)^{i}, i \leqslant k$, удовлетворяют квадратичным соотношениям (2.2), в которых $D_{i}(z)=0$ nрu $i>k$.

Используем теперь это предложение в нашей ситуации. Алгебра $C_{k}$ отображается в решетчатую алгебру с помощью $\varphi(\hbar): d(z) \rightarrow p_{1}^{1}(z)+\hbar p_{2}^{1}(z)+\cdots+$ $\hbar^{k-1} p_{k}^{1}(z)$. При $\hbar \rightarrow 0$ оператор $D_{i}(z)$ стремится к произведению $p_{1}(z) \cdots p_{i}(z)=$ $\widetilde{D}_{i}(z)$. Операторы $D_{i}(z)$ удовлетворяют квадратичным соотношениям, описанным в предложении 2.2, и можно показать, что эти соотношения стремятся к квадратичным соотношениям в алгебре, порожденной операторами $\widetilde{D}_{i}(z)$, которые являются определяющими. Из этого следует, что соотношения, описанные в предложении 2.2, являются определяющими, а потому отображение $\varphi(\hbar)$ является вложением, а также что мы имеем плоское семейство алгебр, зависящее от $\hbar$, и при $\hbar=0$ это алгебра, порожденная вертексными операторами. Все это вместе дает нам доказательство теорем 1.1 и 1.3 при $n=2$.

При больших $n$ будем рассуждать аналогичным образом. Нужно описать операторную алгебру из теоремы 1.1 как квадратично-линейную алгебру, а затем проследить, что происходит с соотношениями в пределе, когда $\hbar \rightarrow 0$.

Предложение 2.3. Алгебра с образующими $a_{i}(z), 1 \leqslant i \leqslant n-1$, u coоmношениями из теоремы 1.1 может быть описана как алгебра с генераторами $A_{i}^{s}(z)=a_{i}(z)^{s}, 1 \leqslant s \leqslant k$, которые удовлетворяют следуюшим соотношениям:

- при фиксированном $i$ операторы $A_{i}^{1}(z), \ldots, A_{i}^{k}(z)$ удовлетворяют соотношениям из предложения 2.2,

- $\left(A_{i}^{s}\right)^{(r)}(z) A_{i+1}^{t}(z)=0$ nри $r<s+t-k$, где $\left(A_{i}^{s}\right)^{(r)}(z)-$ производная порядка $r$ no $z$.

Абелевы вертексные алгебры, которые мы описывали, являются частным случаем следующей конструкции. Пусть задан граф Г без кратных ребер. Предположим, что вершины графа $Г$ можно раскрасить в два цвета (черный и белый), так что каждое ребро соединяет вершины разных цветов. С графом $\Gamma$ мы свяжем последовательность коммутативных вертексных алгебр $\mathscr{O}_{k}(\Gamma)$, $k=1,2, \ldots$ Алгебра $\mathscr{O}_{k}(\Gamma)$ порождена генераторами $a_{\gamma}(z)$, где $\gamma-$ вершины графа, а соотношения имеют вид $a_{\gamma_{1}}(z)^{x} a_{\gamma_{2}}(z)^{y}=0$, если вершины $\gamma_{1}$ и $\gamma_{2}$ соединены ребром и $x+y=k+1$. Очевидно, что алгебра $\mathscr{O}_{k}(\Gamma)$ изоморфна алгебре $\mathscr{O}_{A}$, где $A-$ коммутативная алгебра с понятными соотношениями. Легко видеть, что имеется операция коумножения

$$
\mathscr{O}_{k_{1}+k_{2}}(\Gamma) \rightarrow \mathscr{O}_{k_{1}}(\Gamma) \otimes \mathscr{O}_{k_{2}}(\Gamma), \quad a_{\gamma}(z) \mapsto a_{\gamma}(z) \otimes 1+1 \otimes a_{\gamma}(z)
$$

При $k=1$ алгебра $\mathscr{O}_{1}(\Gamma)$ реализуется как подалгебра в решетчатой вертексоператорной алгебре, $a_{\gamma}(z)$ - вертексные операторы. Построим семейство отображений $\varphi(\hbar)$ из $\mathscr{O}_{k}(\Gamma)$ в $\mathscr{O}_{1}(\Gamma) \otimes \cdots \otimes \mathscr{O}_{1}(\Gamma)(k$ раз $):$ 


$$
\varphi(\hbar) a_{\gamma}(z)=\left\{\begin{array}{r}
a_{\gamma}(z) \otimes 1 \otimes \cdots \otimes 1+\hbar 1 \otimes a_{\gamma}(z) \otimes 1 \otimes \\
\hbar^{k-1} a_{\gamma}(z) \otimes 1 \otimes \cdots \otimes 1+\hbar^{k-2} 1 \otimes a_{\gamma}(z) \otimes 1 \otimes \cdots \otimes 1+\ldots, \\
\gamma-\text { белая вершина, } \\
\gamma-\text { черная вершина. }
\end{array}\right.
$$

В тензорном произведении $\mathscr{O}_{1}(\Gamma) \otimes \cdots \otimes \mathscr{O}_{1}(\Gamma)(k$ раз) получается семейство подалгебр, зависящее от параметра $\hbar$. При $\hbar \rightarrow 0$ алгебра вырождается в квадратичную вертексную алгебру, причем матрица $M$ строится по тому же правилу, что и теоремах 1.3 и 1.4. Заметим, что графы Г в этих двух теоремах - это графы Дынкина, в первом случае для алгебры типа $A_{n}$, во втором случае для аффинной алгебры $A_{n}^{(1)}$. Схема доказательства во всех случаях одна и та же: мы находим в $\mathscr{O}_{k}(\Gamma)$ систему образующих, которые удовлетворяют квадратичнолинейным соотношениям, и показываем, что при $\hbar \rightarrow 0$ эти соотношения вырождаются в соотношения в квадратичной алгебре. Эти рассуждения доказывают, что отображение $\varphi[\hbar]$ при $\hbar \neq 0$ - вложение. Возможно, что этот факт верен для любых графов, а не только для тех, вершины которых можно раскрасить в два цвета. Однако, как доказывать утверждения в общей ситуации, непонятно, поскольку процедура вырождения для общего графа не работает.

\section{§3. Модули демазюровского типа}

Пусть $a_{1}(z), \ldots, a_{n}(z)$ - образующие алгебры $\mathfrak{A}_{1}^{\text {red }}$. Напомним, что соотношения в ней - это $a_{i}(z)^{2}=0, a_{i}(z) a_{i+1}(z)=0$. Пусть $\vec{l}=\left(l_{1}, \ldots, l_{n}\right), l_{1} \leqslant \cdots \leqslant l_{n}$, - вектор с неотрицательными целыми коэффициентами. Обозначим через $R_{\vec{l}}$ представление алгебры $\mathfrak{A}_{1}^{\text {red }}$, индуцированное тривиальным представлением подалгебры $\mathfrak{A}_{1}^{\text {red }}[\vec{l}]$, порожденной Фурье-компонентами $a_{j}[s], 1 \leqslant j \leqslant n, s \geqslant l_{j}$. Пространство $R_{\vec{l}}$ - это представление абелевой алгебры $\mathfrak{a} \otimes \mathbb{C}\left[t, t^{-1}\right]$. Модуль Демазюра $D_{\vec{l}}$ мы определим как $\mathrm{U}(\mathfrak{a} \otimes \mathbb{C}[t])$-подмодуль в $R_{\vec{l}}$, порожденный циклическим вектором. Заметим, что при $n=3 D_{\vec{l}}$ является подпространством в некотором интегрируемом представлении алгебры $\widehat{\mathfrak{s l}}_{3}$ уровня 1 , порожденным экстремальным вектором. Оно совпадает с одним из модулей Демазюра.

Алгебра $\mathfrak{A}_{1}^{\text {red }}$ естественно градуирована $n$-мерной решеткой, и эту градуировку наследует модуль $D_{\vec{l}}$ :

$$
D_{\vec{l}}=\bigoplus_{j_{1}, \ldots, j_{n}} D_{\vec{l}}\left[j_{1}, \ldots, j_{n}\right]
$$

Алгебра $\mathfrak{A}_{1}^{\text {red }}$ имеет еще одну градуировку, по «энергии»: $\operatorname{deg} a_{i}[s]=s, L_{0}-$ coответствующий оператор градуировки. Мы будем считать, что $L_{0}$-градуировка циклического вектора равняется нулю. Величину $\operatorname{tr} q^{L_{0}}$ будем называть $q$-размерностью и обозначать через $\operatorname{dim}_{q}$. Ниже мы используем обозначение $\left[\begin{array}{l}a \\ b\end{array}\right]_{q}$ для
$q$-биномиального коэффициента,

$$
\left[\begin{array}{l}
a \\
b
\end{array}\right]_{q}=\frac{(1-q) \cdots\left(1-q^{a}\right)}{(1-q) \cdots\left(1-q^{a-b}\right)(1-q) \cdots\left(1-q^{b}\right)} .
$$

Предложение 3.1. Имеем

$$
\operatorname{dim}_{q} D_{\vec{l}}\left[j_{1}, \ldots, j_{n}\right]=\left[\begin{array}{l}
l_{1} \\
j_{1}
\end{array}\right]_{q}\left[\begin{array}{c}
l_{2}-j_{1} \\
j_{2}
\end{array}\right]_{q}\left[\begin{array}{c}
l_{3}-j_{2} \\
j_{3}
\end{array}\right]_{q} \ldots\left[\begin{array}{c}
l_{n}-j_{n-1} \\
j_{n}
\end{array}\right]_{q} .
$$


Следующие мономы, примененные к старшему вектору, образуют базис в пространстве $D_{\vec{l}}\left[j_{1}, \ldots, j_{n}\right]$ :

$$
\prod_{m=1}^{n} a_{m}[0]^{\alpha_{0}^{m}} a_{m}[1]^{\alpha_{1}^{m}} \cdots a_{m}\left[l_{m}-j_{m}-1\right]^{\alpha_{l_{m}-j_{m}-1}^{m},} \quad \sum_{\nu} \alpha_{\nu}^{m}=j_{m} .
$$

Доказательство в случае $n=1$ можно найти в работе [1]. В общем случае доказательство почти такое же. Сначала надо показать, что приведенные выше мономы линейно независимы. Для этого удобно выразить токи $a_{i}(z)$ через фермионы. А именно, возьмем $n+1$ токов $\psi_{1}(z), \ldots, \psi_{n+1}(z)$, таких, что $\left[\psi_{i}(z), \psi_{j}(z)\right]_{+}=0$. Токи $\psi_{i}(z)$ порождают операторную алгебру (суперкоммутативную). Алгебра $\mathfrak{A}_{1}^{\text {red }}$ вкладывается в алгебру фермионов: $a_{i}(z) \mapsto$ $\psi_{i}(z) \psi_{i+1}(z)$. Представление $R_{\vec{l}}$ можно реализовать как подпространство в представлении алгебры фермионов, и если выразить наши мономы через фермионы, то видно, что они не могут быть линейно зависимы. Для доказательства того, что мономы действительно образуют базис, нужно оценить размерность модуля $D_{\vec{l}}\left[j_{1}, \ldots, j_{n}\right]$ сверху. Это делается индукцией по $l_{1}, \ldots, l_{n}$.

Заметим, что размерность модуля Демазюра $D_{\vec{l}}\left[j_{1}, \ldots, j_{n}\right]$ разлагается в произведение. Пусть, скажем, $\vec{l}=(l, \ldots, l)$. Тогда $\operatorname{dim} D_{\vec{l}}=\operatorname{dim} D_{(1, \ldots, 1)}^{l}$, а размерность $\operatorname{dim} D_{(1, \ldots, 1)}^{l}$ равна $(n+2)$-му числу Фибоначчи. Модуль Демазюра в нашем случае можно интерпретировать как «фьюжн» (см. [5]), а его характер - как $q$-версию характера тензорного произведения. Точная формулировка этого утверждения такова. Обозначим факторалгебру $\mathbb{C}\left[x_{1}, \ldots, x_{n}\right] /\left(x_{i}^{2}, x_{i} x_{i+1}\right)$ через $C$. Пусть $\theta(u): a_{i} \otimes t^{s} \mapsto x_{i} u^{s}, u \in \mathbb{C},-$ гомоморфизм универсальной обертывающей $\mathrm{U}(\mathfrak{a} \otimes \mathbb{C}[t])$ в $C$. Алгебра $C$ есть свободный модуль над собой; поэтому мы получаем семейство $\mathfrak{a} \otimes \mathbb{C}[t]$-модулей $C(u)$. Тензорное произведение

$$
C\left(u_{1}\right) \otimes \cdots \otimes C\left(u_{l}\right), \quad u_{i} \in \mathbb{C}, u_{i} \neq u_{j} \text { при } i \neq j,
$$

является циклическим $\mathfrak{a} \otimes \mathbb{C}[t]$-модулем, порожденным вектором $1 \otimes \cdots \otimes 1=w$. Оператор $t \partial / \partial t$ определяет градуировку на алгебре $\mathrm{U}(\mathfrak{a} \otimes \mathbb{C}[t]): \mathrm{U}(\mathfrak{a} \otimes \mathbb{C}[t])=$ $U_{0} \oplus U_{1} \oplus \cdots$. Зададим на модуле $C\left(u_{1}\right) \otimes \cdots \otimes C\left(u_{n}\right)$ фильтрацию, положив

$$
F_{j}=\left(U_{0} \oplus U_{1} \oplus \cdots \oplus U_{j}\right) w .
$$

Пусть $\left(C\left(u_{1}\right) \otimes \cdots \otimes C\left(u_{l}\right)\right)^{\mathrm{gr}}-$ присоединенный градуированный модуль. Можно показать, что модули $\left(C\left(u_{1}\right) \otimes \cdots \otimes C\left(u_{l}\right)\right)^{\mathrm{gr}}$ и $\operatorname{dim} D_{(l, \ldots, l)}$ изоморфны.

Пусть $A$ - конечномерная коммутативная алгебра с аугментацией $\varepsilon: A \rightarrow \mathbb{C}$ и $A_{+}-$ядро отображения $\varepsilon$. Для алгебры $\mathscr{O}_{A_{+}}$можно определить модуль Демазюра $D_{l}$ как подпространство в представлении $R_{l}$ алгебры $\mathscr{O}_{A_{+}}$. Здесь $R_{l}$ - представление, индуцированное тривиальным представлением подалгебры $A_{+} \otimes t^{l} \mathbb{C}[t] \subset A_{+} \otimes t^{l} \mathbb{C}\left[t, t^{-1}\right]$, а $D_{l}$ есть $\mathrm{U}\left(A_{+} \otimes \mathbb{C}[t]\right)$-подмодуль в $R_{l}$, порожденный циклическим вектором. Естественно спросить: можно ли модуль $D_{l}$ построить конструкцией «фьюжн», похожей на приведенную выше. В частности, верно ли, что размерность модуля $D_{l}$ равна $(\operatorname{dim} A)^{l}$ ?

Завершим мы этот параграф еще одним примером модуля Демазюра для суперкоммутативной вертексной алгебры. Алгебра $\mathscr{O}$ порождается фермионами $\psi_{1}(z), \ldots, \psi_{n}(z)$, т. е. $\left[\psi_{i}, \psi_{j}\right]_{+}=0$, а соотношения выглядят так: $\psi_{i}(z) \psi_{i}^{\prime}(z)=0$, $\psi_{i}(z) \psi_{i+1}(z)=0$. Соотношения такого рода естественно появляются в теории 
представлений, например, если взять представление уровня один $(N=2)$-супералгебры, то в нем выполняется соотношение $G(z) G^{\prime}(z)=0$, где $G(z)-$ стандартный нечетный ток $(N=2)$-супералгебры.

Модуль Демазюра определяется по той же схеме, т. е. сначала строим модуль $R_{l}$ над алгеброй $\mathscr{O}$, порожденный циклическим вектором $v$, таким, что $\psi_{i}[r] v=0$ при $r \geqslant l$. Определим модуль Демазюра $D_{l}=\mathscr{O}_{\geqslant 0} v$, где $\mathscr{O}_{\geqslant 0}$ порождается компонентами токов $\psi_{i}[s], 1 \leqslant i \leqslant n, s \geqslant 0$. Ясно, что $D_{l}$ - это фактор грассмановой алгебры $\Lambda\left(\psi_{i}[r]\right), 0 \leqslant r \leqslant l, 1 \leqslant i \leqslant n$, по некоторому идеалу. Модуль Демазюра $D_{l}$ можно получить конструкцией, напоминающей «фьюжн». Рассмотрим алгебру $K$ с генераторами $\mu_{i, j}, 1 \leqslant i \leqslant n, 1 \leqslant j \leqslant l$, и соотношениями $\left[\mu_{i, j}, \mu_{i^{\prime}, j^{\prime}}\right]_{+}=0$, а также $\mu_{i+1, j} \mu_{i, j}=0, \mu_{i, j} \mu_{i, j+1}=0$. Базис в такой алгебре нумеруется конфигурациями на прямоугольной $n \times l$-решетке с «отталкиванием». Такая конфигурация - это набор различных вершин решетки, таких, что в конфигурации не встречается двух вершин, соединенных ребром. Фиксируем набор попарно различных комплексных чисел $u_{1}, \ldots, u_{l}$ и зададим гомоморфизм из $\mathscr{O}_{\geqslant 0}$ в $K$, такой, что $\psi_{i}[s] \mapsto \sum_{j=1}^{l} \mu_{i, j} u_{j}^{s}$. Алгебра $\mathscr{O}_{\geqslant 0}$ градуирована, $\operatorname{deg} \psi_{i}[s]=s$; поэтому на $K$ получается фильтрация и присоединенный градуированный модуль - это $D_{l}$. Отметим, что в этом случае можно показать, что результат фьюжн-конструкции не зависит от выбора конфигурации точек $\left\{u_{i}\right\}$. Хорошо известно, что в общем случае это не так.

\section{ЛитЕРАТУРА}

[1] Б. Л. Фейгин, Е. Б. Фейгин, Интегрируемъе $\widehat{s_{2}}$-модули как бесконечные тензорные произведения, в кн.: Фундаментальная математика сегодня, НМУ, М., 2003, 304-334.

[2] B. Feigin, A. Stoyanovski, Quasi-particles models for the representations of Lie algebras and geometry of flag manifold, http://xxx.lanl.gov/abs/hep-th/9308079.

[3] А. В. Стояновский, Б. Л. Фейгин,, Функциональные модели представлений алгебр токов и полубесконечные клетки Шуберта, Функц. анализ и его прил., 28:1 (1994), 68-90.

[4] B. Feigin, E. Frenkel, Coinvariants of nilpotent subalgebras of the Virasoro algebra and partition identities, in: I. M. Gelfand Seminar, Adv. Soviet Math., vol. 16, Part 1, Amer. Math. Soc., Providence, RI, 1993, 139-148.

[5] B. Feigin, S. Loktev, On generalized Kostka polynomials and the quantum Verlinde rule, in: Differential Topology, Infinite-Dimensional Lie Algebras, and Applications, Amer. Math. Soc. Transl. Ser. 2, vol. 194, Amer. Math. Soc., Providence, RI, 1999, 61-79; http://arxiv.org/abs/math/9812093.

[6] I. B. Frenkel, V. G. Kac, Basic representations of affine Lie algebras and dual resonance models, Invent. Math., 62:1 (1980), 23-66.

[7] B. L. Feigin, M. Jimbo, T. Miwa, E. Mukhin, Y. Takeyama, Fermionic formulas for $(k, 3)$-admissible configurations, Publ. Res. Inst. Math. Sci., 40:1 (2004), 125-162.

[8] V. Kac, Vertex Algebras for Beginners, University Lecture Series, vol. 10, Amer. Math. Soc., Providence, RI, 1998.

Национальный исследовательский университет «Высшая школа экономики» 